\title{
Interdisciplinary Research in Public Health: Not quite straightforward
}

Edwin van Teijlingen ${ }^{1-3^{*}}$, Pramod R. Regmi ${ }^{1,4-5}$, Pratik Adhikary ${ }^{6}$, Nirmal Aryal ${ }^{1}$, Padam Simkhada ${ }^{2-3,7}$

${ }^{1}$ Faculty of Health \& Social Sciences, Bournemouth University, UK.

${ }^{2}$ Visiting Professor, Manmohan Memorial Institute of Health Sciences, Kathmandu, Nepal

${ }^{3}$ Visiting Professor, Nobel College, Pokhara University, Nepal

${ }^{4}$ Visiting Research Fellow, Chitwan Medical College, Nepal

${ }^{5}$ Visiting Fellow, DattaMeghe Institute of Medical Sciences, India

${ }^{6}$ Assistant Professor, Manmohan Memorial Institute of Health Sciences, Kathmandu, Nepal

${ }^{7}$ Professor, Liverpool John Moores University, Liverpool, UK

\section{Received:}

1 July 2018

Revised:

13 December 2018

Accepted:

21 January 2019

${ }^{\star}$ Corresponding author

evteijlingen@bour-

nemouth.ac.uk

\begin{abstract}
Over the past few years the practice of disciplines collaborating in interdisciplinary or multidisciplinary research has grown in popularity and not just in the health field. In a multi-faceted discipline such as Public Health, interdisciplinary approaches are a welcome addition to the research field. Interdisciplinary research can bring new insights and understanding across disciplinary boundaries. Novel interdisciplinary research can transcend disciplinary boundaries to address sophisticated and so-called wicked problems in society. However, there are potential difficulties arising from researchers trained in different individual disciplines attempting to work together. We have highlighted some of these issues which interdisciplinary research in Public Health needs to consider and, where necessary, address before they become barriers.
\end{abstract}

Keywords: Multidisciplinarity, interdisciplinarity, cross-disciplinary, transdisciplinary, collaboration

Tweetable abstract: Strengths and potential pitfalls of conducting interdisciplinary Public Health research.

\section{Background}

There is a growing trend towards bringing together different academic disciplines in research into health services, health promotion and public health [1]. There are different approaches which are sometimes used interchangeably, but these approaches are not exactly the same. Interdisciplinary research is slightly different from 'multidisciplinary', 'cross-disciplinary' and 'transdisciplinary' research as outlined in Table 1. Cross-disciplinary is typically the more overarching term and the next three are listed by ascending levels of integration. Multidisciplinary research is simple using two or more disciplinary together perhaps sequentially or in parallel, interdisciplinary is more integrated in its approach and findings and transdisciplinary is the most integrated leading to innovations beyond any specific discipline. For the sake of brevity we shall use the term 'interdisciplinary' approach, since this paper is (a) not about any approach in particular but about the people doing it; and (b) interdisciplinary is probably the most commonly used term. Similarly, in this paper we shall use the broad term 'Public Health' to cover a wide range of sub-disciplines including: 'Health Education \& Promotion', 'Social Policy', 'Health Technology Assessment', 'Health Services Research, 'Epidemiology', 'Health Psychology', or 'Sociology of Health \& Illness'.

Table 1: Mixing disciplines in research

\begin{tabular}{|l|l|}
\hline Approach & Definition\& example \\
\hline Cross-disciplinary & $\begin{array}{l}\text { Relating to more than one discipline, this can be using disciplines alongside each other (i.e. multidisciplinary) to } \\
\text { more integrative approaches (i.e. interdisciplinary or transdisciplinary). }\end{array}$ \\
\hline Multidisciplinary & $\begin{array}{l}\text { Drawing on two or more different disciplines in an additive way to address a common problem as defined within } \\
\text { one discipline. For example, Nursing researchers working with Computing Science colleagues to improve digital } \\
\text { health record systems. }\end{array}$ \\
\hline Interdisciplinary & $\begin{array}{l}\text { Research between fields, addressing a specific topic, integrating insights from (unrelated) disciplines resulting } \\
\text { in higher levels of integration. For example, a collaboration between clinicians, public health researchers, and } \\
\text { statisticians to develop a prevention model. }\end{array}$ \\
\hline Transdisciplinary & $\begin{array}{l}\text { Different disciplines working jointly to create new conceptual, theoretical, methodological, and translational } \\
\text { innovations that integrate and move beyond discipline-specific approaches to address a common problem not } \\
\text { defined in one specific discipline. For example, researchers from different disciplines working together define } \\
\text { and address issues around 'migration health'. }\end{array}$ \\
\hline
\end{tabular}


Interdisciplinary research should not be confused with (a) multi-methods research (mixed-methods); (b) collaborative research, or (c) interdisciplinary methods. Interdisciplinary research has grown somewhat in parallel over the past two to three decades as people began to see the benefits of integrating different research methods into a single health or health services research project. The latter refers to developing new ways of research that cut across disciplinary boundaries [2]. Interdisciplinary research does not necessarily have to involve multiple methods. Of course, different disciplines can collaborate in a study using one single method and, at the same time, within a single project researchers who are all from the same discipline (e.g., all are medical anthropologists or all are nurses or all are epidemiologists) may employ more than one method, i.e. a mixed-methods approach [3].

\section{The interdisciplinary nature of Public Health}

The discipline of Public Health recognises the existence of a range of inter-related causes of ill health in topics such as obesity, infection control, health inequalities, lifestyle factors and social determinants of health. Topics and problems which would often benefit from: (a) complex interventions; and (b) different academic perspectives. We are very well aware that Public Health borrows from and incorporates many other disciplines, as it is a relatively new and still developing academic discipline. For example, people with an undergraduate degree in Public Health by the nature of their training have an interdisciplinary education "because of their knowledge and skills on areas like district health management, research, public health administration, health economics and medical sociology...” [4,p.7]. Focusing on Health Promotion, Tilford noted "the lack of a universal definition of health promotion and likewise the definition of public health, although less contested, is also a developing concept" [5,p.69] and health care are prime topic that can benefit from transdisciplinary research. Because of these characteristics, many agree that Public Health is a discipline where interdisciplinary research would work particularly well [6].

\section{Public Health as an example of interdisciplinary research}

The present emphasis on interdisciplinary research, in many fields not just health, is laudable. However, we would like to remind the reader that this is not an easy option for doing research. Interdisciplinary can be mixed-methods and underpinned by different conflicting (and according to some incommensurable) research philosophies.

Some disciplines are perhaps more open and better suited to interdisciplinary research than others. Public Health is one of these examples that lends itself very well to interdisciplinary research as its constituent disciplines use all common methods, from primary to secondary research and from qualitative to quantitative and mixed-methods research. Public Health researchers commonly use multi-methods approaches comprising both primary, using for example, surveys, interviews, focus group discussions, and secondary research, using large routine databases and systematic reviews. And within such mixed-methods research it employs a range of qualitative, quantitative and mixed-methods approaches [3]. Public Health is probably the most interdisciplinary discipline as it brings together many sub-specialities, from Epidemiology to Health Economics and from Health Psychology to Sociology of Health \& Illness and from Health Management to Medical Statistics. In addition, Public Health researcher and practitioners are often collaborating and supporting with clinical disciplines. Whatever discipline interests you most, an interdisciplinary approach can enrich your learning because of its emphasis on using multiple fields of ideas, inquiry, and methods of research. For researchers addressing important questions and issues, one set of methods to analyzing and understanding is probably not sufficient on its own. Chances are that working in Public Health your chosen approach is already richly interdisciplinary.

Thus several conditions need to be fulfilled to ensure interdisciplinary research has the greatest chance of succeeding. Importantly, for true interdisciplinary research we need to consider the following key factors: (a) a common goal and shared vision; (b) regular assessment of progress; (c) active leadership; (d) allocation of individual roles and contributions; (e) study plan; and (f) regular communication and constructive comments among team members [7].

\section{The ideal interdisciplinary researcher}

Based on years of planning, conducting and writing up research we are highlighting here some of the key issues around interdisciplinary research. We reflected on questions such as: "What does it mean to us to be interdisciplinary researchers?" and "What quality does an interdisciplinary researcher need over and above those working as a researcher in a single discipline?" We, the five authors, all see ourselves as Public Health researchers, but we have very different educational backgrounds and training, reflecting the diversity and interdisciplinarity in the field. Several of us have a first degree in Education or Health Education, but one has a first degree in Sociology. Whilst four of the five authors have Master degrees in Public Health and/or Health Promotion, two have a Master in Education. Most of us have a PhD. in Public Health, but again one of us has a PhD. in Sociology. Issues of interdisciplinary team working often add to the general strains of team working. Team working itself can be a very challenging aspect of work.

\section{Why does it matter?}

First of all, most people in Public Health across the globe are still trained at university with disciplines. Although the world is changing, we argue that you need the foundation knowledge of your discipline to anchor yourself. You have to know your basic Sociology, Health Management, Epidemiology or Nursing, etc. before you can embark on research that brings together different disciplines. Disciplines have (slightly) different ways of working, in how the discipline typically approaches problems, the jargon it uses and the solutions it tends to put forward. For example, Health Economics, which is based on economic principles, is a common subject for any Public Health de- 
gree because it offers advice for funding among the competing priorities. As a member of a discipline, you need to present your work reflecting your discipline's style and expectations; this often affects how you write and where you publish.

Secondly, not all interdisciplinary research is the same. For example, in one study midwives and obstetricians, both health professionals, may work together whilst in a different study an economist may work with a paediatrician. One can envisage different views and preconceptions and approaches occurring in the latter collaboration than in the former. And that is not to say that two health professionals from different disciplines working together will be any easier.

\section{Teaching}

In terms of education and training, subject specialists, be it Health Economists, Medical Statisticians or Health Psychologists, are required to use their knowledge of structural aspects of their discipline to teach nursing students one day and physiotherapists another day. Thus on the same day a Medical Sociologist may be teaching medical students about the theoretical background of doctor-patient interactions from a Feminist perspective, and have enough knowledge of Social Psychology to run a workshop for midwifery students on Communication Skills. Two days later they might be teaching Content Analysis on a research methods course for Sport Studies students or an introduction to SPSS to Physiotherapy students. Not to forget the odd lecture on the importance of Qualitative Methods to a number of different audiences, ranging from the newly appointed members to the local medical research ethics committee, the Medical School postgraduate support group or the Specialist Registrars in Training to become Public Health doctors. An additional benefit is that because generalists teach such a wide range of topics to such a wide range of audiences, they are perhaps more likely to have thought about the nature of their own teaching and their students' learning.

\section{Research}

On the research side topic specialists face equally challenging demands on their range of skills and knowledge. They are required to have a broad knowledge at hand to represent, for example the social science perspective to a study of a disease they know little about, designed by clinicians with a health services research outcome in mind.

In terms of research methods Public Health researchers find that they have to be versed in both qualitative and quantitative methods. Working multidisciplinary or interdisciplinary means that they have to be able to understand the methods of the epidemiologists (e.g. 'interrupted-time series' or 'nested-case control studies') and those health service researchers (e.g. 'double-blind randomised controlled trials') and have the whole range of qualitative methods at hand to improve the quality of the overall study. Conversely, they have to be able to add a quantitative aspect to a focus group study designed by a research nurse, conduct the statistical analysis and report the findings.

Who leads in interdisciplinary team? We may make the analogy between different partners in an interdisciplinary PhD. study. There is a lead supervisor, but this is not always the same person during the life time of the $\mathrm{PhD}$. Three of the authors have been in supervisory teams where the clinician starts off as the lead supervisor, during the questionnaire design it becomes more and more the sociologist and during the analysis the lead supervisor is really the statistician and at the end during the thesis write up phase can be any of them.

\section{Interdisciplinary publishing}

Some of our specialist colleagues highly appreciate generalists rather than single-minded discipline specialists. Unfortunately, the academic world still does not reward the generalist as much as the specialist, in terms of both promotion and career opportunities. Many of interdisciplinary researchers in our field would still call themselves a psychologist or a nurse or an economist working in Public Health. This is important when it comes to dissemination. Following the growth of interdisciplinary research and working we have seen the growth of 'specialist' interdisciplinary journals, see for example: Digital Health, or Health \& Place or Health. These offer an obvious outlet for interdisciplinary papers, but many partners in an interdisciplinary team still prefer to publish in their own disciplinary journals. This is understandable as discipline specific journals have a longer history and hence more likely to have a higher reputation and impact factor.

We must also remember that different disciplines have different publishing traditions. For example, in an interdisciplinary team comprising a Social Geographer, a Health Economist and a Public Health doctor, different expectations may exist. On the one hand, in geography it appears that field workers who collected the data are included as authors irrespective of whether they contributed to the analysis or writing process [8]. Whilst, in most health journals there is a clear understanding that authorship credit should be based only on substantial contribution to the data analysis and interpretation, the drafting and editing of the article and approval of the final manuscript [9]. At the same time the Public Health doctor and the social geographer probably expect the author order to be determined by the amount of contribution to the paper a co-author has made, with the author who has done most of the work being the first author. But this is done differently in the discipline of Health Economics where co-authors might be listed in alphabetical order [8].

\section{Conclusions}

In Public Health research the terms multidisciplinary and interdisciplinary are often used interchangeably, but these are not the same (see Table 1). Often these concepts are linked to notions of mixed-methods research, which, as we have highlighted, is again something different. We need to consider how we will train (at least some) truly interdisciplinary Ph.D. students to help us deal with the complex questions of the 21st century. This is a challenge in many countries as a lot of higher education at universities and colleges is still largely 
discipline based. Inter-disciplinary writing 'crossover', interdisciplinary articles or even novel methodological approaches help strengthen studies that cross the boundaries of the research academic disciplines.

\section{References}

1. Choi BCK, Pak AWP (2007). Multidisciplinarity, interdisciplinarity, and transdisciplinarity in health research, services, education and policy: 2. Promotors, barriers and strategies of enhancement, Clin Invest Med, 30(6):E224-E232.

2. Hesse-Biber S. (2016) Doing Interdisciplinary Mixed Methods Health Care Research: Working the Boundaries, Tensions, and Synergistic Potential of Team-Based Research. Qual Health Res 26: 649-658.

3. MacKenzie Bryers H, van Teijlingen E,Pitchforth E. (2014) Advocating mixed-methods approaches in health research, Nepal J Epidemiol4(5): 417-422. http://www. nepjol.info/index.php/NJE/article/view/12018/9768

4. Khanal P, Mishra SR. (2014) Exploring Opportunities for Public Health Graduates in Government Health System in Nepal. Health Prospect: J Publ Health $13(1)$ : 7-11.

5. Tilford S. (2018) Using a health promotion lens, Int J Health Prom Educ 56: 68-69.

6. Green J, Thorogood N (2018) Qualitative Methods for Health Research (4th edn), London: Sage.

7. Nair KM, Dolovich L, Brazil K, Raina P. (2008) It's all about relationships: A qualitative study of health researchers' perspectives of conducting interdisciplinary health research, BMC Health Serv Res, 8:110. Available: https://bmchealthservres.biomedcentral.com/articles/10.1186/1472-6963-8-110 [Accessed 5 September 2018 ]

8. Hundley V, van Teijlingen E, Simkhada P. (2013) Academic authorship: who, why and in what order? Health Renaissance 11: 98-101. Available: www.healthrenaissance. org.np/uploads/Download/vol-11-2/Page_99_101_Editorial.pdf [Accessed 22December 2018 ]

9. International Committee of Medical Journal Editors (ICMJE) Defining the Role of Authors and Contributors. Available: http://www.icmje.org/recommendations/ browse/roles-and-responsibilities/defining-the-role-of-authors-and-contributors.html\#two [Accessed 10 November 2018] 